\title{
Formation Processes of Zinc Excimer Thin Films Due to Ion-Recombination Processes
}

\section{—Excited States of Zinc with "Permanent Life Time"}

\author{
Li Chen*, Shinichiro Yamashita, Mitsugi Hamasaki, Hirotaka Manaka, Kozo Obara \\ Graduate School of Science and Engineering, Kagoshima University, Kagoshima, Japan \\ Email: ${ }^{*}$ chenli2009@live.jp
}

Received 9 February 2014; revised 9 March 2014; accepted 17 March 2014

Copyright (C) 2014 by authors and Scientific Research Publishing Inc.

This work is licensed under the Creative Commons Attribution International License (CC BY).

http://creativecommons.org/licenses/by/4.0/

(c) (i) Open Access

\begin{abstract}
In materials science, the number of $d$-electrons of transition metals is an essentially important factor controlling characteristics of alloys and compounds. In this paper, we show an example to control the number of d-electrons (holes) by using inner-core electron excitation of zinc atoms. An important feature of our research is that we can make a long lifetime excited electronic state of zinc $\left(3 d^{8}\right)$, and the life-time of excited zinc is more than 307 days. At first, the experimental apparatus and boundary conditions of the ion-recombination processes were explained. From results of XPS, excited zinc films showed satellites peaks what caused by the final state of $3 \mathrm{~d}^{8}$ and the charge transfer final state of $3 \mathbf{d}^{10} \underline{L}^{2}$. Excited states of zinc were formatted at the surface of substrate caused by ion-recombination process between $\mathrm{Zn}^{+}$and $\mathrm{Zn}^{-}$. The excited zinc diffused from substrate surface to the surface of the excited zinc thin film. Intensity of excited zinc is proportional to the intensity of electron on the substrate.
\end{abstract}

\section{Keywords}

Zinc Excimer, Ion-Recombination, Zn3d8, Charge Transfer, XPS

\section{Introduction}

Technique in materials chemistry is due to control of the most outer shell electron. From the standpoint of materials chemistry, study of new materials' creation under the control of the inner shell electron is scarce. Control of inner-shell electron is involved in the excitation process and the relaxation process. About these processes, there are a lot of researches. The excimer in the excited state is derived from excited dimmer for short. Excimer is the electron excited states atoms and molecules in combination with other atoms and molecules. The lifetime of an

"Corresponding author.

How to cite this paper: Chen, L., Yamashita, S., Hamasaki, M., Manaka, H. and Obara, K. (2014) Formation Processes of Zinc Excimer Thin Films Due to Ion-recombination Processes. Journal of Applied Mathematics and Physics, 2, 449-456.

http://dx.doi.org/10.4236/jamp.2014.27055 
excimer is very short, on the order of nanoseconds. The binding of a larger number of excited atoms forms Rydberg matter clusters, the lifetime of which can exceed many seconds [1]. As we all know, the transition mental group has special unfulfilled 3d electronic configuration. And zinc $\left(\Pi_{b}\right)$ is the only fulfilled 3d electronic configuration of transition metal elements. So, our group is focused on creating permanent lifetime zinc excimer, learning the mechanism formation processes and analyzing the resulted phenomenon. The excitation can be exploited in modification of the surface layers, modification of the bulk in a selected way, energy transport and charge transport, energy storage and so on. The chemical reactions depend on the number of outer electrons usually; however, if we can arrange the inner-core electron system of the atom, we can get a new periodic table, that is, a new development platform [2].

\section{Experiment Apparatus}

The experiment apparatus which was developed just by our research group is an integral evaporation system with transmission electron spectroscopy evaluation (Electron-assisted PVD) [3] [4]. The conceptual diagram of vacuum experimental system and experiments condition were shown in Figure 1. The vacuum system ultimate pressure was decreased to $10^{-5} \mathrm{~Pa}$. The emitted thermal electrons from the tungsten filament hairpin were considered as the incident electron source. Thermal electrons emitted from the electron gun be accelerated by the bias voltage $\left(\mathrm{V}_{\mathrm{B}}=0 \sim 240 \mathrm{~V}\right)$, what applied to the substrate electrode, and thermal electrons irradiate a wide range to the substrate surface. The incident angle of electrons was 45 from the substrate surface, Then the $0.1 \mathrm{~g}$ zinc atoms were deposited on the insulate area from the effusion cell at $600^{\circ} \mathrm{C}$, and the insulate area is measured about diameter is $6.5 \mathrm{~mm}$ at the center of the sapphire substrate.

\subsection{The Reaction Field}

Figure 2 is a conceptual diagram of the reaction field [3]. From Figure 1 and Figure 2, at the peripheral portion of the oxide aluminum substrate, equipped with a gold film in order to provide a bias voltage of the incident electrons. When the bias voltage added from electron gun (cathode) to gold thin film (anode), the reaction field became an electric field and vector of this electric field is from gold thin film to electron gun. Clone force $\mathbf{F}$ acting on the point charge is write by $\mathbf{F}=\mathrm{QE}$ [5] [6], at first, acting a clone force from electron gun toward gold thin film to the incident electron, with the sapphire substrate, the incident electron can adhere on the sapphire. Then, electrons have been accumulating on top of the substrate (Adhesion electrons create a potential/field at the substrate surface.), number of adhered electrons have been increasing until potential of the substrate surface to $\mathrm{V}_{\mathrm{B}}$. Now, a stable and downward electric field has been created by adhered electrons. Initial velocity of incident zinc particles is downward. Zinc particles only have momentum in the vertical direction. The sapphire surface was electrified by incident electrons up to the same magnitude of potential as the anode bias. This growth field makes selective growth field for $\mathrm{Zn}^{+}$.

Electrons form a stable and substrate orientation electric field at the center of oxide aluminum substrate where radius $\mathrm{R}=3.5 \mathrm{~mm}$. Corresponds to thermal electrons emitted from the electron gun be accelerated by the bias



Figure 1. Vacuum experimental system and condition. 


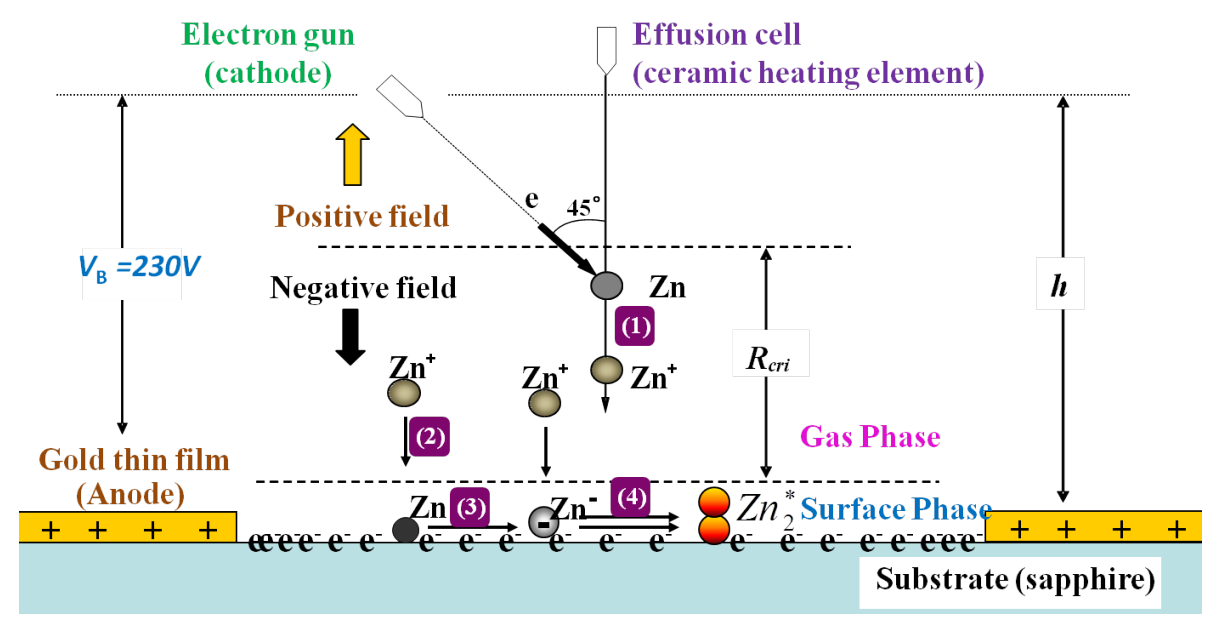

Figure 2. Reaction field of the experiment.

voltage $\left(V_{B}=0 \sim 240 \mathrm{~V}\right)$, the potential of the insulating portion is negative and its absolute value is equal to the bias potential $\mathrm{V}_{\mathrm{B}}$. Corresponds to the bias potential, the electrons that in the reaction field have a stability energy and it equal total of potential energy and kinetic energy $\left(\mathbf{E}_{\mathbf{e}}=\mathbf{E}_{\mathbf{p}}+\mathbf{E}_{\mathbf{k}}\right)$ [6]. And the electrons on the substrate must have a potential energy of $0 \sim 240 \mathrm{eV}$. In other words, incident electronic energy should be $0 \sim 240 \mathrm{eV}$. By the quantum theory, the possible energy value of atoms is discrete. Then, when the transition to the (excited state) high energy state from low energy state, just excitation energy of discrete energy difference $\Delta E=E_{2}-E_{1}$ is required [7].

\subsection{Ion-Recombination Process}

In a vapor phase growth processes, kinetic energy of incident atoms in the gas phase were dissipated at the surface phase in the condensation processes [8]. Zinc excimers were formed on sapphire substrates within diameter of $6.5 \mathrm{~mm}$ area, where enclosed by gold electrode, which is the anode for incident electrons exciting zinc atoms. An estimate excited process of zinc thin films included 4 steps:

Charging process:

Ionization:

$$
\mathrm{Zn}+\mathrm{e}_{(K . E)}^{-} \rightarrow \mathrm{Zn}^{+}+2 \mathrm{e}^{-}
$$

Electron Attachment:

$$
\begin{aligned}
& \mathrm{Zn}^{+}+\mathrm{e}^{-} \rightarrow \mathrm{Zn} \\
& \mathrm{Zn}+\mathrm{e}^{-} \rightarrow \mathrm{Zn}^{-}
\end{aligned}
$$

Ion-Recombination:

$$
\mathrm{Zn}^{+}+\mathrm{Zn}^{-} \rightarrow\left(\mathrm{Zn}^{*}\right)_{2}
$$

Firstly, zinc that incidence from effusion cell is iodinated by incidence electron in the negative field (1) in the gas phase, lifetime of $\mathrm{Zn}^{+}$can be extended by the electric field [9]; Secondly, the iodinated $\mathrm{Zn}^{+}$adhered to the substrate (surface of sapphire), and adhered $\mathrm{Zn}^{+}$combined with electron to $\mathrm{Zn}$ at substrate surface (2); Thirdly, $\mathrm{Zn}$ combined with electron to $\mathrm{Zn}^{-}$at substrate (3). Lastly, $\mathrm{Zn}^{-}$what adhered at substrate combined with $\mathrm{Zn}^{+}$what come from negative field and excited state formed (4). Lifetime of negative ions is much shorter than positive ions [10]. An electron charged up sapphire surface was used to elongate the lifetime of $\mathrm{Zn}^{-}$. Normally, lifetime of excimer is transitory, counting as ns, but excited zinc atoms are from surface phase fixed in solid phase at the first period, so that, the excited states were preserved for a long time. We daring assumed that the excited zinc was created though the special charging field due to ion-recombination processes. Electron density distribution determined the density of excited zinc. 


\subsection{Surface Electron Distribution}

From equations, excited zinc depends on the electrons that adhered on the substrate. The substrate surface is charged by incident electrons. The electron density and distance between electrons on substrate should be [3] [6]:

$$
\sigma(r)=\frac{2 \varepsilon_{0} V}{e\left(R^{2}-r^{2}\right)^{1 / 2}}
$$

Firstly, the sapphire surface was electrified by incident electrons up to the same magnitude of potential as the anode bias. We know that the bias potential is a fixed number from $0 \mathrm{~V}$ to $240 \mathrm{~V}$. From Equation (5), to keep the surface potential constant, the surface electron density must depend on the radius. The electron density at edge area is much higher than at the center, on other hand, distance between electrons at edge area is smaller than at the center. Excited zinc depended on electron intensity, so that excited zinc growth from edge. Furthermore, distance between excited zinc at edge area is smaller than that at the center, because excited zinc depended on electron. From Equation (5), the intensity of excited zinc depended on the surface electron intensity. The zinc atoms were deposited on the insulative area, which measured $3.0 \sim 7.0 \mathrm{~mm}$ in diameter at the center of the substrate. Improved to trial, the reaction field of the experiments could be decreased to micrometer. The electron distribution was simulated in Figure 3.

\section{Results and Discussions}

\subsection{Results of XPS}

In Figure 4(a), XPS spectrum of Zn 3d, 3p, 3s, 2p, C1s and O1s of excited zinc thin films were compared between 7 days' sample and 307 days' sample. 7 days, what means the sample was detected after it was made 7 days. 307 days, what means the same sample was detected after it was made 307 days. The core level photoelectron spectrum shape of Zn 2d, 3p, 3s, 2p in excited zinc film were shown in Figure 4(a) and Figure 4(b). The standard point of these data is C1s $(285.0 \mathrm{eV})$. The sample shown in Figure 4 was deposited at $230 \mathrm{eV}$. The same sample was measured after 7 days and 307 days. Figure 4(a) C1s and O1s peaks of 7 days were extremely week. Very small amounts of $\mathrm{O}$ and $\mathrm{C}$ were adsorbed. After 300 days, amounts of $\mathrm{O}$ and $\mathrm{C}$ were adsorbed at the excited film, and there are some chemical reactions.

The finer detail binding energies of mental zinc: $\mathrm{L}_{2} 2 \mathrm{p}_{1 / 2}, \mathrm{~L}_{3} 2 \mathrm{p}_{3 / 2}, \mathrm{M}_{1} 3 \mathrm{~s}, \mathrm{M}_{2} 3 \mathrm{p}_{1 / 2}, \mathrm{M}_{3} 3 \mathrm{p}_{3 / 2}, \mathrm{M}_{4} 3 \mathrm{~d}_{3 / 2}, \mathrm{M}_{5} 3 \mathrm{~d}_{5 / 2}$ at $1044.8 \mathrm{eV}, 1021.7 \mathrm{eV}, 139.6 \mathrm{eV}, 91.4 \mathrm{eV}, 88.8 \mathrm{eV}, 10.1 \mathrm{eV}$, and $\mathrm{C} 1 \mathrm{~s}$ is $285.1 \mathrm{eV}$ [11]. Figures 4-7days showed binding energy of zinc similar to normal zinc. Figures 4-7days only showed general characteristics of zinc. Figure 4-307days showed the special characteristics of zinc spectrum. Binding energy of $2 \mathrm{p} 1 / 2$ is $23.1 \mathrm{eV}$ $(1044.9-1021.8=23.1)$ higher than $2 \mathrm{p} 3 / 2$. Binding energy of 3p1/2 is $2.8 \mathrm{eV}(91.4-88.6=2.8)$ higher than $3 \mathrm{p} 3 / 2$. Binding energy of $3 \mathrm{~d} 3 / 2$ is $0.5 \mathrm{eV}$ (10.1 - $9.6=0.5$ ) higher than $3 \mathrm{~d} 3 / 2$. Spectrum shape of Zn 3d, 3p, 3s and $2 p$ in Figures 4-7days showed similar features of mental zinc. On other hand, Spectrum shape of Zn 3d, 3p, $3 s$ and 2p in Figure 4-307days showed new features. The satellite peaks at high binding energy side hadn't been reported ever. There are some peaks at $15 \mathrm{eV}$ and about $3.6 \mathrm{eV}$ high binding energy side of the Figure 4(b)-307days, but there is smooth at $15 \mathrm{eV}$ high binding energy side of the Figure 4(b)-7days.

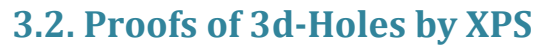

Figure 5 showed decomposition of inner-shell photoelectron spectroscopy of Figures 3-7days. First of all, we fitted out the curves of 3d, 3p, 3s and 2p3/2 by Gaussian functions. Fitted peaks curves lined in green, the backgrounds were cut by the green point lines, and peak sum showed in pink curves. $3 \mathrm{~d}: 3 \mathrm{~d}$ was fitted to 2 nearly peaks, peak center was $0 \mathrm{eV}$ and $1.0 \mathrm{eV}$. 3d specific included final states of $3 \mathrm{~d} 5 / 2$ and $3 \mathrm{~d} 3 / 2$, binding energy of $3 \mathrm{~d} 5 / 2$ and $3 \mathrm{~d} 3 / 2$ is approaching. 3p:3p also was fitted to 2 peaks, and the binding energy of peak center was 2.6 $\mathrm{eV}$ and $0 \mathrm{eV}$. 3p specific included 3p1/2 and 3p3/2. 3s:3s was fitted to a single Gaussian function peak at $0 \mathrm{eV}$. $2 \mathrm{p} 3 / 2: 2 \mathrm{p} 3 / 2$ also was fitted to a single Gaussian function peak at $0 \mathrm{eV}$. Refer to 3.1 and 3.2, the spectrum of zinc showed normal zinc features. On the upper surface of excited zinc film, there wasn't excited zinc existed after it was made 7 days.

Figure 6 showed decomposition of inner-shell photoelectron spectroscopy of Figure 3-307days. First of all, we considering that, Figure 5 included main peaks at $0 \mathrm{eV}$ as Figure 4 and shift peaks at the high binding ener- 


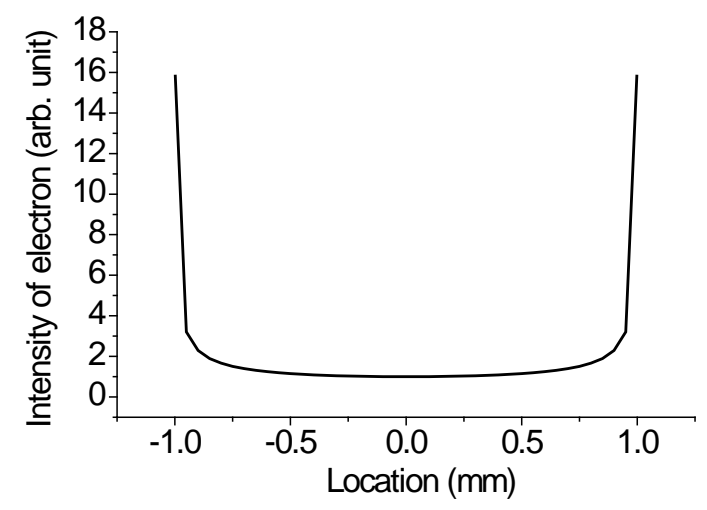

Figure 3. Surface electron distribution.

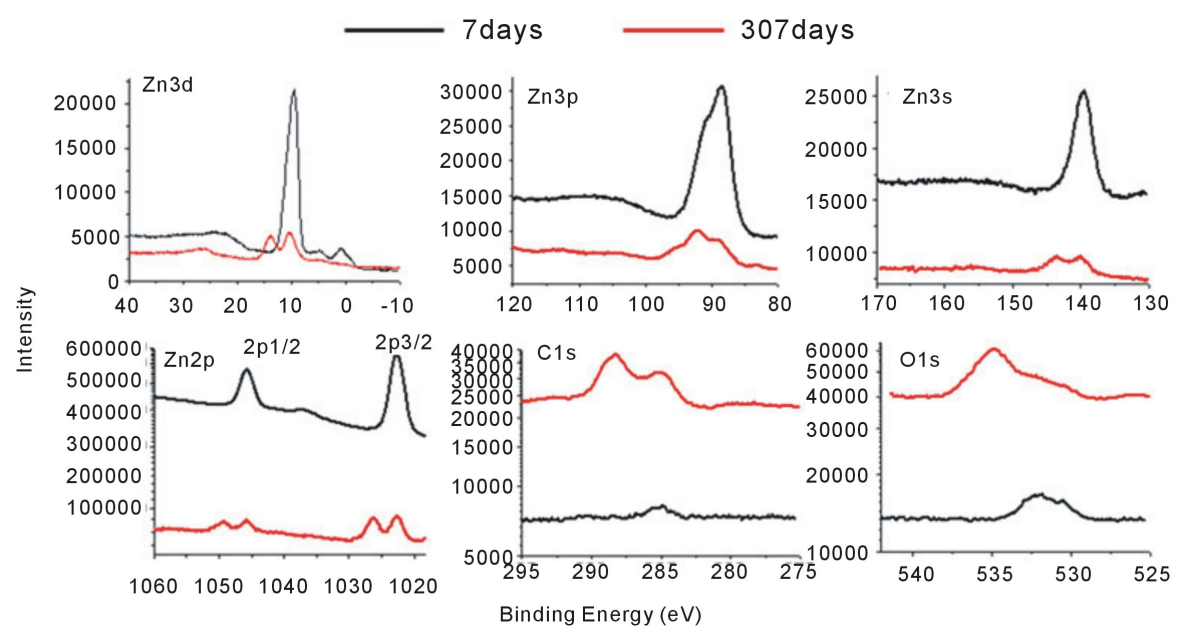

(a)

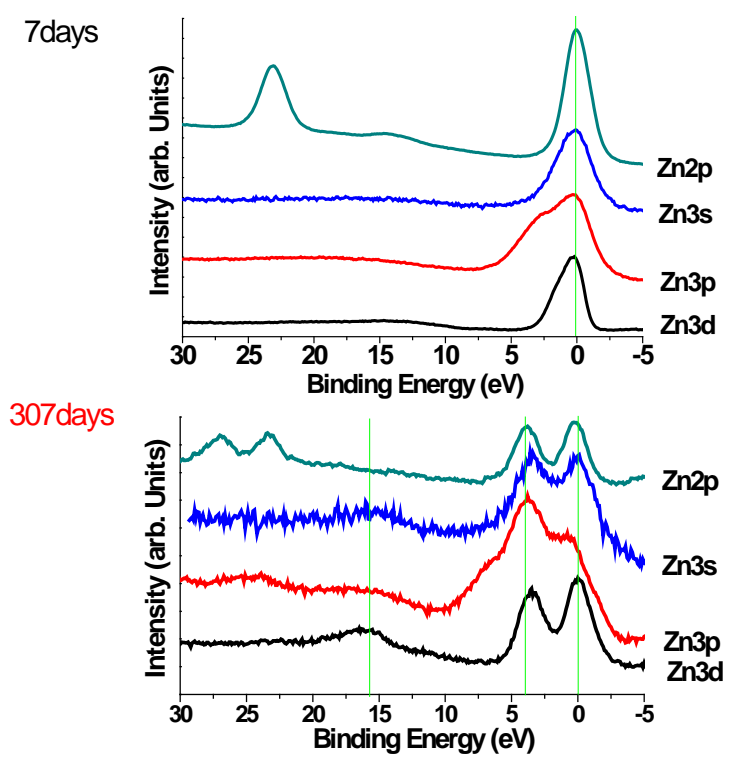

(b)

Figure 4. (a) XPS spectrum of Zn3d, 3p, 3s, 2p, C1s and O1s of excited zinc thin films, what has made 7 days and 307 days before. (b) XPS spectrum of Zn3d, 3p, 3s and 2p of excited zinc thin films was composed between 7 days and 307 days. 


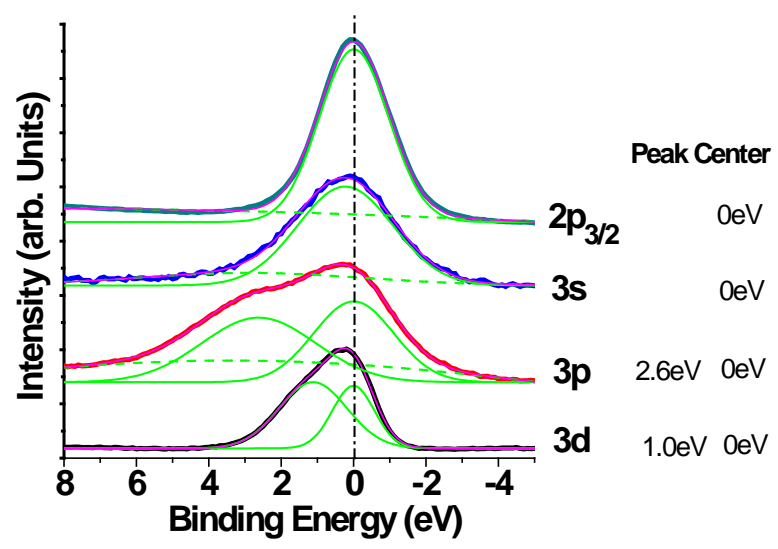

Figure 5. Ingredient decomposition of XPS spectrum of Zn 3d, $3 p$, 3s and 2p3/2 of Figures 3-7days.

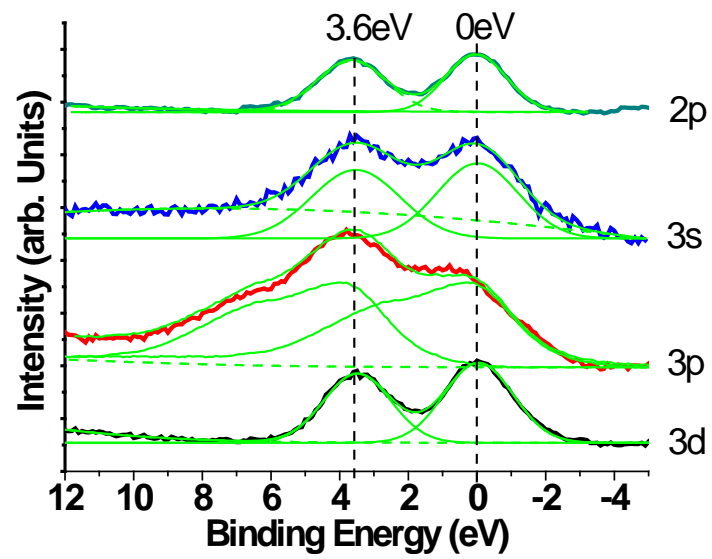

Figure 6. Ingredient decomposition of XPS spectrum of Zn 3d, 3p, 3s and 2p3/2 of Figure 4(b)-307days.

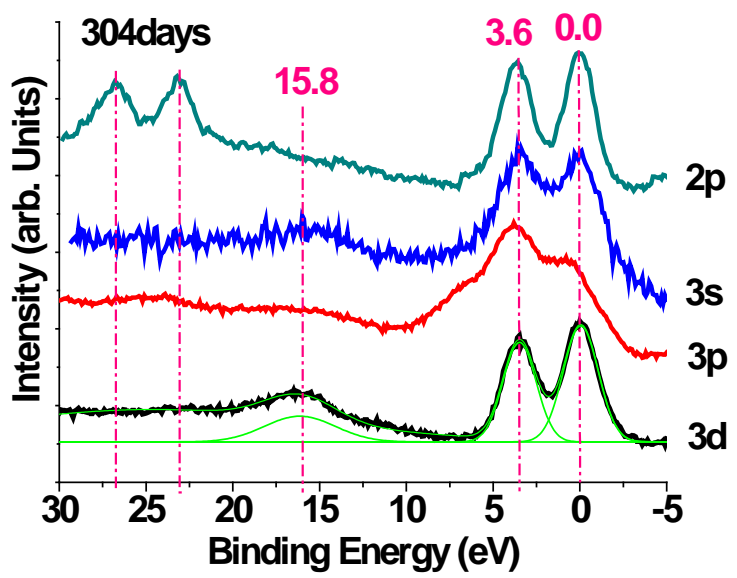

Figure 7. XPS spectrum of excited zinc thin films at 3d, $3 p, 3 s$ and 2p.

gy side. Refer to Figure 4, fitted out the curves of 3d, 3p, 3s and 2p3/2 by Gaussian functions, and the backgrounds were cut by the green point lines. $3 \mathrm{~d}$ :3d specific included final states of $3 \mathrm{~d} 5 / 2$ and $3 \mathrm{~d} 3 / 2$, and binding 
energy of $3 d 5 / 2$ and $3 d 3 / 2$ is approaching, that it couldn`t be fitted out. 3d was fitted to 2 peaks, peak center was $0 \mathrm{eV}$ and $3.6 \mathrm{eV}$, corresponding to main $3 \mathrm{~d}$ peak of zinc and $+3.6 \mathrm{eV}$ shift peak. 3p:3p should be fitted to four peaks, corresponding to two of main 3p peak and two of shifted peaks of main 3p peak at high binding energy side. It was fitted to 2 curves, corresponding to main 3p peak of zinc and $+3.6 \mathrm{eV}$ shift peak. 3s:3s was fitted to two single Gaussian function peaks at $0 \mathrm{eV}$ and $+3.6 \mathrm{eV}$. 2p3/2:2p3/2 also was fitted to a single Gaussian function peaks at $0 \mathrm{eV}$ and $+3.6 \mathrm{eV}$. There are two kinds of zinc atoms on the film.

We referred the theory of reference [3]. The data of the transition metals from $\mathrm{Mn}^{2+}$ to $\mathrm{Zn}^{2+}$, exhibit satellites effects which may be qualitatively interpreted in this way [12]. Structures for ions with almost filled shell for $\mathrm{Cu}^{2+} 3 \mathrm{~d}^{9}, \mathrm{Ni}^{2+} 3 \mathrm{~d}^{8}$, and $\mathrm{Co}^{2+} 3 \mathrm{~d}^{7}$; and neither effect for the filled shell in $\mathrm{Zn}^{2+} 3 \mathrm{~d}^{10}$. The resolved satellites are believed to be due to inner hole of $3 \mathrm{~d}^{\mathrm{n}}(\mathrm{n} \leq 9)$. Means if there has no inner hole of $3 \mathrm{~d}$ state, there has not satellite peaks. In Figure 5, the spectrum of zinc showed normal zinc features and no normal zinc features. The peaks of

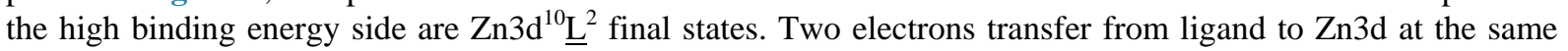
site [13] [14]. The charge transfer energy is $3.6 \mathrm{eV}$. The ligand elements only considered to pollution of $\mathrm{C}$ or $\mathrm{O}$ atoms in air.

\subsection{Proofs of Long Lifetime $3 \mathrm{~d}-$ Holes by XPS}

Spectrum shape of Zn 3d, 3p, 3s and 2p in Figure 6 showed new features. Three binding energies were observed at $0 \mathrm{eV}, 3.6 \mathrm{eV}$ and $15.8 \mathrm{eV}$. The peaks at $0 \mathrm{eV}, 3.6 \mathrm{eV}$ and $15.8 \mathrm{eV}$ are normal zinc atom $3 \mathrm{~d}^{10}$, charge transferred $3 \mathrm{~d}^{10} \underline{\mathrm{L}}^{2}$ and $3 \mathrm{~d}^{8}$. The components that form the sharp rise of the low binding energy side originated to $\mathrm{d}^{10}$ final state $\left(3 \mathrm{~d}^{10}\right)$; The peak of the high binding energy side was identified as the peak due to $\mathrm{d}^{8}$ final state and movement of the hole did not occur $\left(3 \mathrm{~d}^{8}\right)$; Between the $3 \mathrm{~d}^{10}$ and $3 \mathrm{~d}^{8}$, there is a satellite peak, in response to the coulomb force of the inner shell hole, the hole in the valence band has moved from the Zn3d orbital to the same site ligand orbital was $\mathrm{d}^{10} \underline{\mathrm{L}}^{2}$ final state $\left(3 \mathrm{~d}^{10} \underline{L}^{2}\right)$. This process is charge transfer (CT) and the ligand should be $\mathrm{O}$ or C [15] [16]. On the special excited zinc thin film (Figure 6), the peaks were observed at $0 \mathrm{eV}, 3.6 \mathrm{eV}$ and $15.8 \mathrm{eV}$ corresponds to the $3 \mathrm{~d}^{10}, 3 \mathrm{~d}^{10} \underline{L}^{2}$ and $3 \mathrm{~d}^{8}$. The charge transfer $\Delta=3.6 \mathrm{eV}$. The coulomb repulsion between 3d electrons of zinc $\mathrm{U}_{\mathrm{dd}}=15.8 / 2=7.9 \mathrm{eV}$. Excited zinc formatted at the bottom of the excited thin films. The excited zinc will automatically spread to the surface of the zinc film. The diffusion mechanism is not yet understood. The lifetime of excited is more than 307 days.

\section{Conclusion}

The formation processes of $\mathrm{Zn}$ excimer exactly depend on discreet energies which are combinations of binding energies of electrons of $\mathrm{Zn}$ atom. The condensed states depend on the excitation processes of each ion. Intensity of the condensed states depends on intensity of electrons. And the intensity of electrons depends on location and bias voltage. We present formation processes of excited 3d states of $\mathrm{Zn}$ atoms with abnormally long-lifetime. The lifetime of excited states can calculate by days (more than 307 days) and our samples can be re-excited. The excited zinc atoms form excimers (excited molecule) due to ion-recombination process what happed in the surface phase. In the special zinc thin film, excited state of zinc, $3 \mathrm{~d}^{8}$ was observed. The charge transfer $\Delta=3.6 \mathrm{eV}$ and ligand was considered to $\mathrm{C} / \mathrm{O}$. Excited zinc formatted at the bottom of the excited thin films. The excited zinc will automatically spread to the surface of the zinc film. Excimer is exploited in energy transport and storage becomes possible.

\section{References}

[1] Birks, J.B. (1975) Excimers. Reports on Progress in Physics, 38, 903-974. http://dx.doi.org/10.1088/0034-4885/38/8/001

[2] Noriaki Itoh, A. and Stoneham, M. (2001) Materials Modification by Electronic Excitation.

[3] Hamasaki, M., Obara, M. and Obara, K. (2011) AIP Conference Proceedings, 1415, 43-50. http://dx.doi.org/10.1063/1.3667216

[4] Donald, M.M. (1998) Handbook of Physical Vapor Deposition (PVD) Processing.

[5] Morrison Jr., F.A. (1969) Ind. Engineering Chemistry Fundamentals, 8, 594-595. http://dx.doi.org/10.1021/i160031a041

[6] Watanabe, Y. and Aoyagi, A. (2004) Electromagnetism. 
[7] Koidesyou, I. (2010) Quantum Mechanics.

[8] Ehrlich, G. and Hudda, G. (1966) Atomic View of Surface Self-Diffusion: Tungsten on Tungsten. The Journal of Chemical Physics, 44, 1039-1049. http://dx.doi.org/10.1063/1.1726787

[9] Gerassimou, D.E. and Rapakoulial, D.E. (1987) Excite Ion Lifetime for the Electric Field Measurement in the Sheath Region.

[10] Herzberg, G. (2007) Atomic Spectra and Atomic Structure. 229-230.

[11] Gunnar, S. (1973) Journal of Electron Spectroscopy and Related Phenomena, 2, 75-86.

[12] Rosencwaig, A., Wertheim, G.K. and Guggenheim, H.J. (1971) Origins of Satellites on Inner-Shell Photoelectron Spectra. Physical Review Letters, 27, 479. http://dx.doi.org/10.1103/PhysRevLett.27.479

[13] Brunschwig, B.S., Carol, C. and Norman, S. (1998) Coordination Chemistry Reviews, 177, 61-79. http://dx.doi.org/10.1016/S0010-8545(98)00188-X

[14] Ma, Y., Ma, Y.G., Zhang, H.Y., Shen, J.C. and Che, C.M. (1998) Synthetic Metals, 94, 245-248. http://dx.doi.org/10.1016/S0379-6779(97)04166-0

[15] de Groot, F. and Kotani, A. (2008) Core Level Spectroscopy of Solids.

[16] Kowalczyk, S.P., Pollak, R.A., McFeely, F.R., Lwy, L. and Shirley, D.A. (1973) L2, 3M45M45 Auger Spectra of Metallic Copper and Zinc: Theory and Experiment. Physical Review B, 8, 2387. http://dx.doi.org/10.1103/PhysRevB.8.2387 